\title{
El caldo de cultivo para un pacto social. Algunos datos para reformar el mercado de trabajo en España
}

\author{
The breeding ground for a social pact. Some data \\ to reform the labor market in Spain
}

\author{
Daniel Pérez del Prado \\ Profesor Titular Ac. De Derecho del Trabajo y de la Seguridad Social \\ Universidad Carlos III de Madrid \\ ORCID ID: 0000-0001-7106-6769
}

doi: https://doi.org/10.20318/labos.2021.6051

\section{Introducción. El punto de arranque: reflexionar y actuar.}

Decía Keynes que la dificultad no reside en el desarrollo de nuevas ideas, sino en escapar de las viejas. La magnitud tanto del drama social que atravesamos, por la concurrencia de dos crisis económicas prácticamente seguidas, además de la sanitaria; como de la transformación económica y social que la digitalización implica, no solamente hace que esta reflexión esté más de actualidad que nunca, sino que nos exige ir incluso más allá. Necesitamos efectivamente escapar de las viejas ideas para alcanzar nuevas más brillantes e innovadoras, pero, sobre todo, necesitamos también poderlas poner en práctica cuanto antes.

En este sentido debemos dar la bienvenida a todo intento, no solamente de generar debate, sino especialmente de propiciar nuevas líneas de actuación y de reforma. En estas mismas páginas, los profesores Cabeza y Cardona han dedicado un artículo a desarrollar algunas ideas que ya habían esbozado previamente acerca de cómo debiera reformarse el conjunto del Derecho social, pues sus reflexiones van más allá del Derecho del Trabajo para alcanzar también al de la Seguridad Social y al Derecho del Empleo.

Su punto de partida es que, con la conformación del nuevo Gobierno y el profundo proceso de digitalización y de los efectos que de ella se derivan, se abre un nuevo campo de acción que debiera conducirnos a alcanzar un nuevo pacto social. Dicho de otra forma, los procesos que sufrimos son tan profundos y la necesidad de cambio tan urgente, que las medidas que se necesitan serían de la suficiente magnitud como para que debieran ser acordadas por consenso, no solamente de los agentes sociales, sino del conjunto de la sociedad. Este en este contexto, la llegada de la pandemia y de la crisis económica que la acompańa no habrían hecho más que agudizar tanto la necesidad de cambio como lo perentorio de su introducción.

En el texto, de forma sencilla, concisa y razonada, se van delineando los diferentes campos de actuación que a su juicio son necesarias y que en buena medida implican revisitar gran parte de los problemas tradicionales de nuestro mercado de trabajo que, a pesar de su gravedad, han terminado por enquistarse. Tal es el caso de la contratación temporal y de la dualidad que de la misma se deriva, de la estructura de la negociación colectiva o de la articulación de las políticas de empleo. Aun así,

"Este trabajo forma parte del proyecto de investigación "Cambio tecnológico y transformación de las fuentes laborales: Ley y convenio colectivo ante la disrupción digital” (RTI2018-094547-B-C21). 
también han tenido tiempo los autores de analizar algunas otras cuestiones resurgidas al calor de los debates más recientes, como el papel que el autoempleo ha de tener en nuestro mercado de trabajo, que resucita gracias al debate en torno a la laboralidad de los riders; o sobre el crecimiento de los salarios que, como veremos, trasciende del efecto coyuntural que sobre los mismos tuvo la Gran Recesión.

Pero además de señalar las áreas de interés, el texto es eminentemente propositivo. Los autores no solamente hacen un esfuerzo por estudiar cuál es la concreta situación en cada una de las áreas señaladas, sino por apuntar posibles soluciones de mejora. No podía ser de otra forma dada la magnitud de los problemas a que se refieren y de los cambios que se sugieren. Como acabamos de señalar, buena parte de las ineficiencias detectadas vienen de antiguo, por lo que han sido suficientemente analizadas. Es tiempo, por tanto, de pasar a la acción, no solamente porque el debate a alcanzado un grado de madurez suficiente, sino porque las necesidades sociales a que se refieren son perentorias.

El reto no es menor, tanto por la magnitud de los problemas, como por la necesidad de articular ya propuestas de actuación. A ello habría que añadir una dificultad más, precisamente la que nos advertía Keynes, liberarnos de nuestras viejas ideas, para formular nuevas radicalmente novedosas y eficaces.

El presente estudio pretende acompañar el mencionado artículo y complementar, no el trabajo, que no lo necesita, sino el debate que se propone suscitar. En las páginas que siguen abordaremos de forma muy sintética y desde una perspectiva eminentemente cuantitativa cada una de las áreas de reforma indicadas en él, aportando datos adicionales para el análisis y algunos otros argumentos para el debate. Para ello, a la presente introducción, le seguirán los correspondientes apartados dedicados a la contratación temporal, el empleo autónomo, la estructura de la negociación colectiva, la recuperación de los salarios y la configuración de las políticas de empleo. Finalmente, se cerrará con unas brevísimas conclusiones.

\section{A vueltas con la contratación temporal}

Que la alta temporalidad es uno de los grandes problemas de nuestro mercado de trabajo está fuera de dudas. Que los esfuerzos que hemos hecho para intentar corregirla han dado escasos frutos, también.

En efecto, es cierto que hemos logrado pasar, por decirlo de una forma gráfica, de niveles excesivamente altos, cercanos al 33\% (como muestra el Gráfico 2, en el período 2002-2006), a simplemente muy altos, del entorno del $26 \%$ (en el mismo gráfico, evitando el efecto coyuntural de las crisis, el lapso que iría de 2014 al primer trimestre de 2020), pero esto no ha impedido que, como refleja el Gráfico 1, sigamos a la cabeza de Europa en esta materia, prácticamente duplicando la media del conjunto de la Unión y de la eurozona, con 4,5 puntos más que el segundo, Portugal.

Estas altas y persistentes tasas de temporalidad son negativas tanto en términos de equidad como de eficiencia económica ${ }^{1}$. Por lo que respecta a lo primero, aunque la contratación temporal ha venido siendo utilizada desde los 80 como una forma de política de empleo, lo cierto es que los estudios no han conseguido demostrar que el fomento de la temporalidad genere más puestos de trabajo $^{2}$. Es verdad que se observa un efecto positivo en el momento de tránsito de un sistema rígido a otro flexible, del que seguramente saldría beneficiado nuestro país en su momento ${ }^{3}$, permitiendo una salida más rápida, desde el punto de vista del empleo, de las crisis económicas; pero también que las altas tasas de rotación que produce suelen llevar aparejado una fortísima volatilidad en empleo.

${ }^{1}$ Dolado, J. J., Jimeno, J. F., “Contratación temporal y costes de despido en España: Lecciones para el futuro desde la perspectiva del pasado", cit., pág. 5 .

${ }^{2}$ Bentolila, S.; Saint-Paul, G., "The macroeconomic impact of flexible labor contracts, with an application to Spain", CEMFI, DT, № $9106,1991$.

${ }^{3}$ Ibid. 


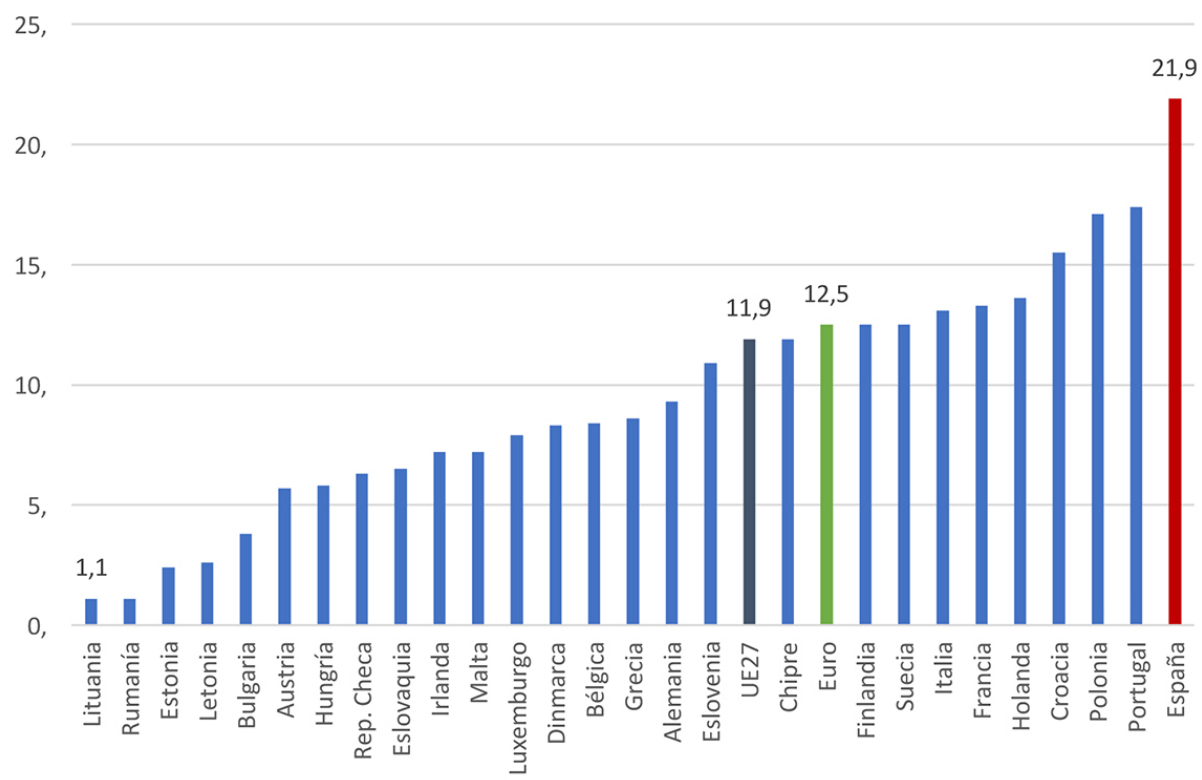

Gráfico 1. Tasa de temporalidad UE.

Fuente: elaboración propia a partir de los datos del Eurostat. 2019.

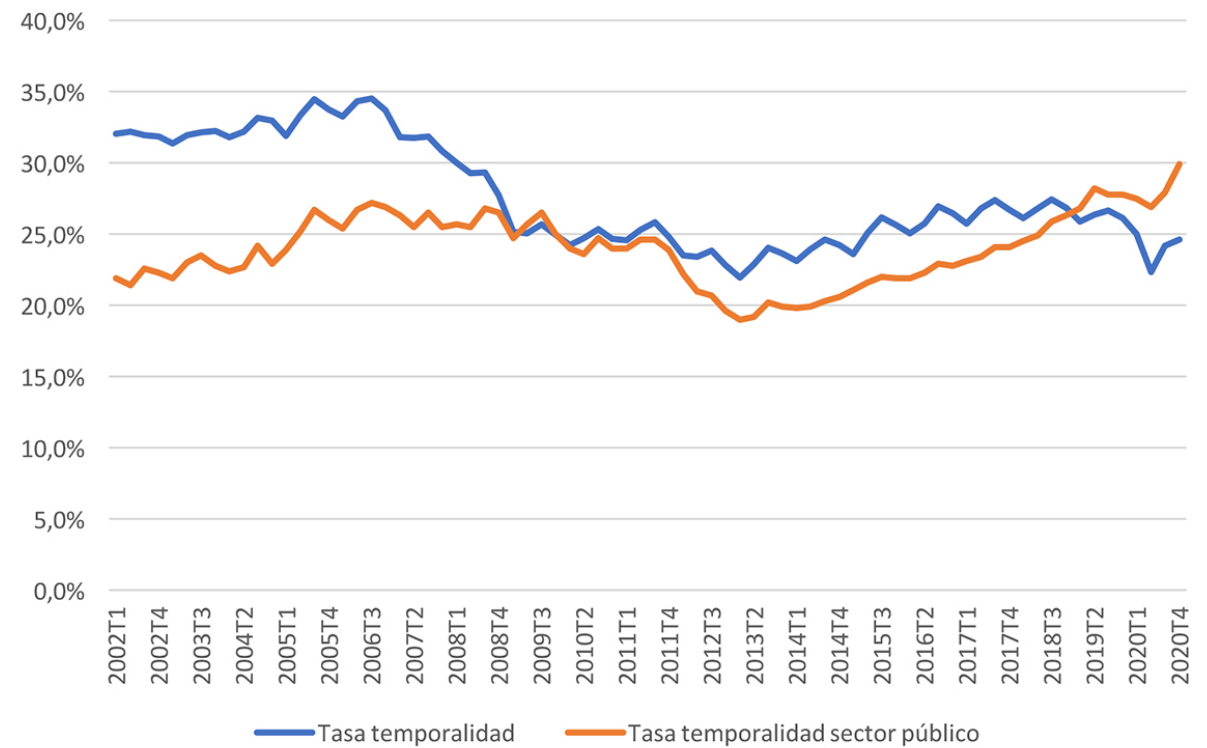

Gráfico 2. Evolución tasa de temporalidad.

Fuente: elaboración propia a partir de los datos del INE.

Por lo que hace a la equidad, la evidencia empírica demuestra que la contratación temporal lleva aparejada una remuneración más baja ${ }^{4}$, una menor inversión en capital humano específico para trabajadores con una antigüedad superior a los tres ańos y una mayor probabilidad de sufrir un

${ }^{4}$ De La Rica, S., "La penalización salarial de la temporalidad: ¿Qué efectos tiene en las decisiones familiares?”, en Propuesta para la reactivación laboral en España, Fedea, 2010. J. F. Jimeno; L. Toharia, "The effects of fixed-term employment on wages: theory and evidence from Spain", Investigaciones Economicas, vol. 17, 3, 1993, Fundación SEPI, p. 489. Un resultado similar, cifrando tal diferencia en torno al 10\%, se alcanza en Hernanz, V.; El trabajo temporal y la segmentación: un estudio de las transiciones laborales, Consejo Económico y Social, 2003, págs. 177-189. 


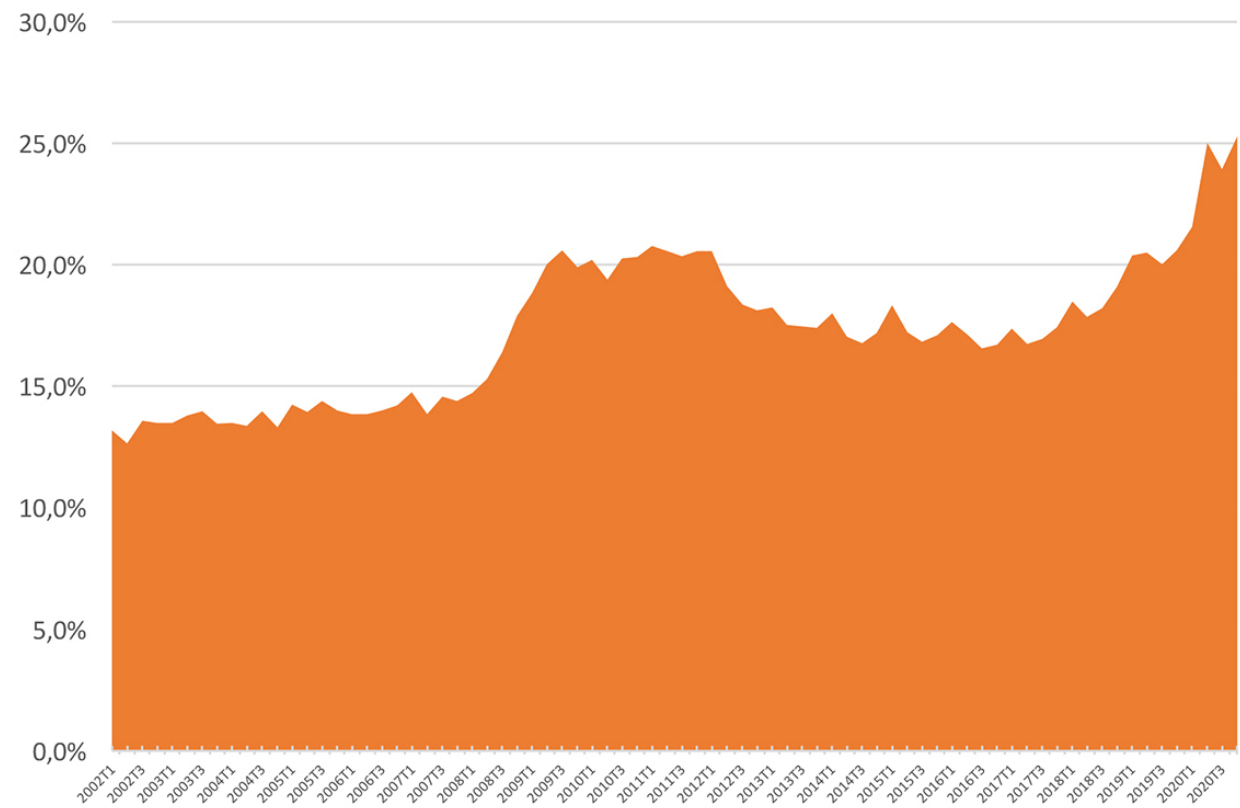

Gráfico 3. \% Asalariados temporales del sector público respecto del total de asalariados temporales. Fuente: elaboración propia a partir de los datos del INE.

accidente de trabajo ${ }^{5}$, si bien esto no tiene que ver tanto con el tipo de contratación como con las actividades en que se concentra la temporalidad. Además, sus efectos trascienden lo laboral. La excesiva temporalidad de nuestro mercado de trabajo estaría incidiendo en decisiones vitales de las personas trabajadoras como la de la convivencia en común o el momento de entrada en la maternidad ${ }^{6}$.

Del panorama descrito se deduce que se son necesarias acciones en todos los ámbitos posibles, el de la extinción, el de la cotización, el del control y la sanción, no solamente porque las medidas parezcan haber producido un impacto reducido, sino porque junto con las medidas que se tomaran es preciso también transmitir un mensaje claro y contundente a la sociedad en general y a nuestro tejido productivo en particular.

De ahí la importancia también que tiene la incorporación de la Administración en esa estrategia de acción. Como puede comprobarse en el Gráfico 2, la tasa de temporalidad en las administraciones públicas supera ya a la del conjunto de los asalariados en más de cinco puntos. A mediados de 2020, las CCAA fueron la Administración que más contribuyó a esta situación, con una tasa de temporalidad del 31,9\%, seguida de la local, con un 17,8\% y, a mayor distancia, las empresas públicas, con un 17,8\%. Solo la Administración General del Estado y de la Seguridad Social mantuvieron unos niveles aceptables, del 9,5\% y 9,1\% respectivamente ${ }^{7}$, muy próximo a los objetivos marcados en II Acuerdo Gobierno-Sindicatos para la mejora del empleo público y las condiciones de trabajo. Por su parte, el Gráfico 3 muestra muy a las claras el terrible afecto que tuvo la crisis financiera sobre la temporalidad del sector público. El peso del sector público en el conjunto de la temporalidad pasó de representar menos del $15 \%$ a más del $20 \%$. La recuperación no solamente no

${ }^{5}$ Durán, F., Informe sobre los riesgos laborales y su prevención. La seguridad y salud en el trabajo en España, Presidencia del Gobierno, Madrid, 2001; Herranz Martín, V.; Toharia Cortés, L., "Do temporary contracts increase work accidents? A microeconomic comparison between Italy and Spain", Fedea DT, N ${ }^{o}$ 10, 2003.

${ }^{6}$ Un análisis de los efectos de la temporalidad en todos estos ámbitos puede encontrarse en Pérez del Prado, D., "Los efectos de la temporalidad más allá del mercado de trabajo", en Gómez Abelleira, F. J. (ed.) La temporalidad laboral como problema jurídico: diagnósticos y soluciones, Tirant lo Blanch, 2014, pp. 61-96.

${ }^{7}$ Asempleo, El monográfico del Mercado Laboral, Asempleo, 2020, fecha de consulta 15 febrero 2021, en https://asempleo.com/ wp-content/uploads/2020/10/Bolet\%C3\%ADn-AML-n\%C2\%BA-159-Octubre-2020-monografico.pdf. 
ha reducido esta tendencia, sino que la ha incrementado. A finales de 2020, una cuarta parte de los asalariados temporales de nuestro país trabajaban para una Administración pública.

En suma, la Administración parece haberse convertido en parte del problema. Pero su contribución a la solución no radica tanto en el ámbito cuantitativo, porque se logre reducir los alarmantes niveles de temporalidad alcanzados, como cualitativos, porque apunte en la dirección anteriormente indicada, la de transmitir que desde todos los ámbitos se ha de batallar por poner fin a los problemas derivados de la excesiva temporalidad.

\section{El aparente resurgir del empleo autónomo}

Por otra parte, el debate en torno a la laboralidad de los riders o, en general, del trabajo en plataformas, ha propiciado que prestemos una mayor atención al trabajo por cuenta propia. Con independencia de cómo evolucione este debate, lo cierto es que lo más probable es que no haya una única solución, sino varias en función de las plataformas ${ }^{8}$ e, incluso, varias para una única plataforma, simplemente porque adapte su estrategia a las resoluciones judiciales que se vayan dictando.

Aunque el peso del trabajo en plataformas es reducido en relación con el conjunto de la ocupación?, ha tenido la virtud de volver nuestra mirada hacia el trabajo autónomo. Al margen de este concreto sector, es necesario seguir avanzando en la mejora de las condiciones de trabajo y de protección social de esta forma de prestación de servicios, relativamente importante en nuestro país. Como puede comprobarse en el siguiente gráfico, en España el trabajo autónomo supone el 15\% del total, apenas un 1\% más que la media europea. Además, parece que la crisis económica le ha impactado en menor medida, pues solamente se ha reducido un punto porcentual en comparación con los niveles registrados antes de la Gran Recesión.

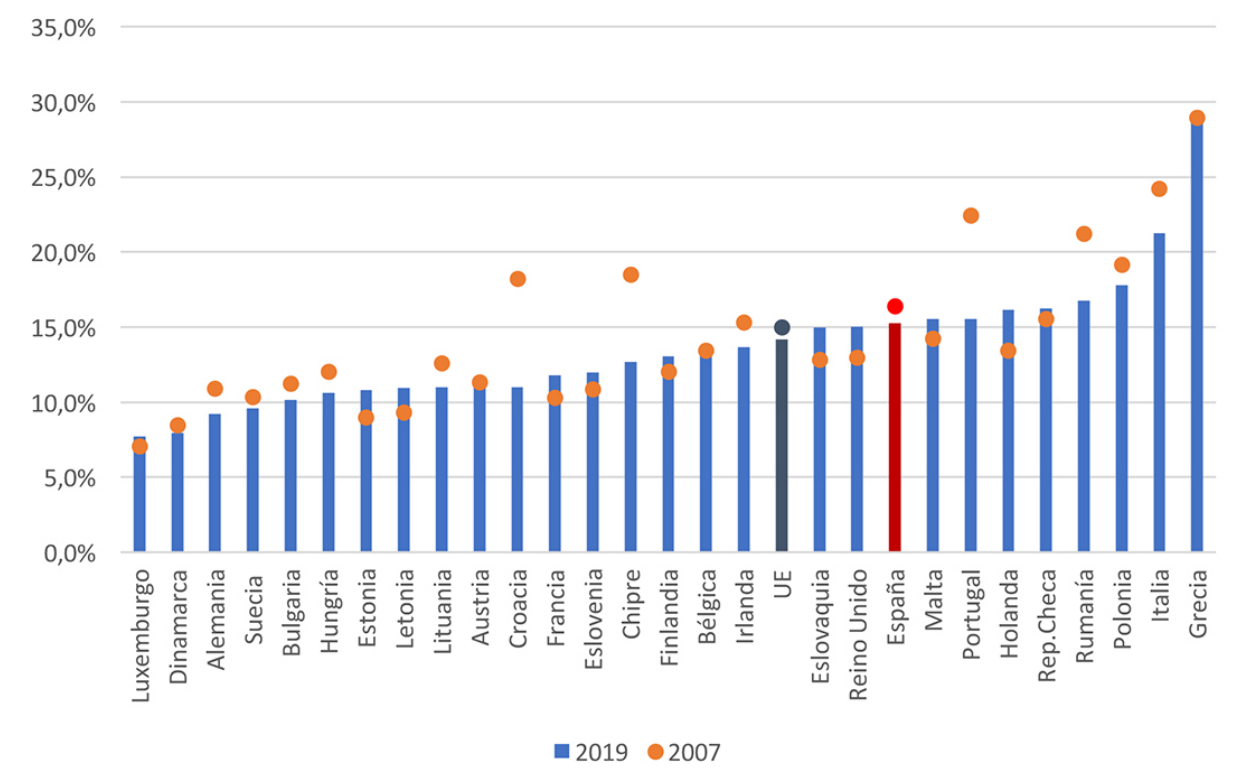

Gráfico 4. \% Trabajo autónomo respecto del total.

Fuente: elaboración propia a partir de los datos del Eurostat.

\footnotetext{
${ }^{8}$ Mercader Uguina, J. R., El futuro del trabajo en la era de la digitalización y la robótica, Tirant lo Blanch, Valencia, 2017.

${ }^{9}$ Urzí Brancati, M.C; Pesole, A; Fernández-Macías, E., New evidence on platform workers in Europe: results from the second COLLEEM survey., European Commission, Directorate-General for Economic and Financial Affairs, Brussels, 2020.
} 
Si vamos al detalle de esta cifra podemos observar, tal y como muestra el gráfico siguiente, que más del $60 \%$ de los autónomos son personas físicas, frente al $30 \%$ que presta servicios bajo la cobertura de algún tipo de sociedad. Destaca también en el gráfico el reducidísimo papel que tienen los TRADES, el 0,25\%, mucho menos que los cálculos que se hicieron cuando se creó la figura y muy probablemente menos de los que haya en la realidad.

Sea como fuere, estos datos evidencian, por una parte, el fracaso que ha supuesto esta figura intermedia, al menos en lo que respecta a su afloramiento; por otra, el enorme riesgo con el que se emprende en nuestro país, pues son una minoría los que optan por iniciar una actividad autónoma bajo la cobertura de una sociedad o colectivamente ${ }^{10}$. Este es un aspecto sumamente importante, especialmente si se tiene en cuenta que, de acuerdo con los datos de Eurostat ${ }^{11}$, en torno a la mitad de los trabajadores autónomos fracasan a los tres años de iniciar un proyecto profesional.

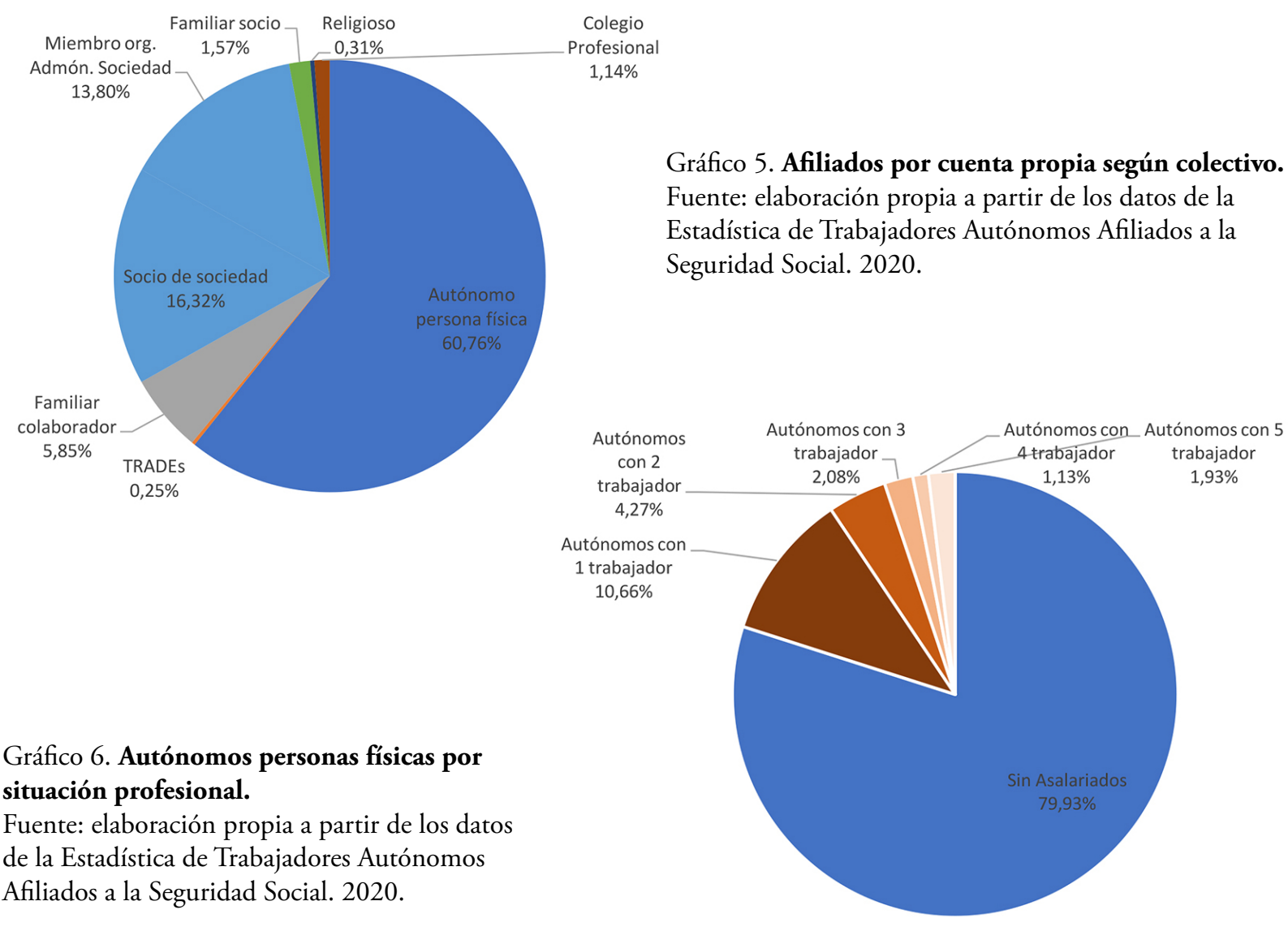

En este sentido, los datos también contribuyen a desmitificar la imagen del emprendimiento como motor generador de empleo. Por una parte, ya hemos visto el peso relativo que tiene respecto del conjunto de la ocupación. Por otra, el Gráfico 6 evidencia que en torno al $80 \%$ de los autónomos no cuenta con personas trabajadoras a su cargo, cifra que se eleva hasta el $91 \%$ si ańadimos a los que tiene a una sola persona. Dicho de otra forma, las cifras ponen de manifiesto que el emprendimiento no es ni la fórmula mágica para generar el propio empleo, ni el de terceros.

\footnotetext{
${ }^{10} \mathrm{Al}$ margen de la fórmula societaria, lo cierto es que cuando se emprenden colectivamente se reparten los costes, la inversión y, por consiguiente, también los riesgos.

${ }^{11}$ https://ec.europa.eu/eurostat/statistics-explained/index.php/Business_demography_statistics
} 
Al margen de lo anterior, este último gráfico también pone de manifiesto el enorme margen de maniobra con que contamos para mejorar las condiciones de trabajo y de protección social de este colectivo. En efecto, el Parlamento Europeo ha mostrado que los autónomos sin asalariados tienen un mayor riesgo de precariedad en comparación con aquellos con asalariados ${ }^{12}$. Por su parte, la Comisión también está trabajando en una iniciativa para mejorar las condiciones laborales de las personas que prestan servicios a través de plataformas, como se anunció en el Programa de trabajo de la Comisión para 2021. En el marco de estas actuaciones, ha lanzado una consulta pública ${ }^{13}$ para sortear las limitaciones que el art. 101 TFUE, relativo a la libre competencia en el seno del mercado interior, impone a una hipotética negociación colectiva de este colectivo. Pues bien, esta excepción pivota precisamente sobre la persona trabajadora autónoma sin empleados a su cargo que, como vemos, son la inmensa mayoría.

La negociación colectiva puede ser por tanto un instrumento muy eficaz para la mejora de las condiciones de trabajo de este colectivo, superando las debilidades que hasta ahora han planteado los acuerdos de interés profesional. De cómo se aborde ese debate y, en su caso, se lleve a efecto, dependerá en buena medida su éxito ${ }^{14}$.

\section{IV. ¿Una negociación colectiva pendular?}

Pero la negociación colectiva en su sentido clásico reviste notables problemas, cuyo máximo exponente es el inacabado debate en torno a su estructura ${ }^{15}$. Si bien la descentralización de la negociación colectiva ha sido uno de los objetivos más importantes y comunes en la regulación del mercado laboral entre las economías occidentales ${ }^{16}$, los resultados alcanzados en la mayor parte de ellos han sido muy reducidos.

Nuestro país no es una excepción, ni en lo que hace al debate, ni en lo que respecta a sus frutos. En relación con lo primero, la reforma laboral de 2012 marca el último hito y hasta ahora el más importante en la tendencia descrita. En un contexto económico y social sumamente complicado, se hacía necesario adoptar decisiones arriesgas y, sin lugar a dudas, que la reforma así lo hizo. «Era necesario, por tanto, avanzar y ofrecer nuevas soluciones. La Ley 3/2012 [...] es un paso decidido en esa dirección en lo que se refiere a la función de la negociación colectiva como instrumento de regulación del sistema de relaciones laborales» ${ }^{17}$.

Sin embargo, a pesar de lo novedoso y audaz de las decisiones tomadas, hoy, casi una década después, los resultados, al menos en lo que hace a los cambios en la estructura de la negociación co-

\footnotetext{
${ }^{12}$ European Parliament, Precarious Employment in Europe: Patterns, Trends and Policy Strategies, European, Brussels, 2017.

${ }^{13} \mathrm{https} / /$ ec.europa.eu/info/law/better-regulation/have-your-say/initiatives/12483-Collective-bargaining-agreements-for-self-employed-scope-of-application-EU-competition-rules. Sobre las dificultades que plantea la regulación española, Nieto RojAs, P., "Acción colectiva en las plataformas digitales. ¿̇indicatos tradicionales y movimientos de base para representar idénticos intereses?", en $E l$ futuro del trabajo: cien años de la OIT. XXIX Congreso Anual de la Asociación Española de Derecho del Trabajo y de la Seguridad Social, Ministerio de Trabajo y Seguridad Social, Madrid, 2019 (Informes y estudios), pp. 1499-1516.

${ }^{14}$ Sobre la extensión de algunos elementos propios del trabajo de cuenta ajena al trabajo por cuenta propia, véase RoDRíGUEZ Fernández, M. L., "Calificación jurídica de la relación que une a los prestadores de servicios con las plataformas digitales", en Plataformas digitales y mercado de trabajo, Subdirección General de Información Administrativa y Publicaciones, 2018, pp. 57-89.

${ }^{15}$ Mercader Uguina, J. R., Estructura de la negociación colectiva y relaciones entre convenios, Editorial Civitas : Ediciones de la Universidad Autónoma de Madrid, Madrid, 1994.

${ }^{16}$ OECD, COLLECTIVE BARGAINING IN A CHANGING WORLD OF WORK, OECD, Geneva, 2017, pp. 125-186, fecha de consulta 17 febrero 2021, en https://www.oecd-ilibrary.org/employment/oecd-employment-outlook-2017/collective-bargaining-in-a-changingworld-of-work_empl_outlook-2017-8-en.

${ }^{17}$ Mercader Uguina, J. R., "La empresa como nuevo centro de gravedad de la estructura de la negociación colectiva: la reforma de la Ley 3/2012", en García-Perrote Escartín, I., Mercader Uguina, J. R. (eds.) La regulación del mercado laboral: un análisis de la Ley 3/2012 y de los aspectos laborales del Real Decreto-ley 20/2012), Lex Nova, Valladolid, 2012, p. 420.
} 


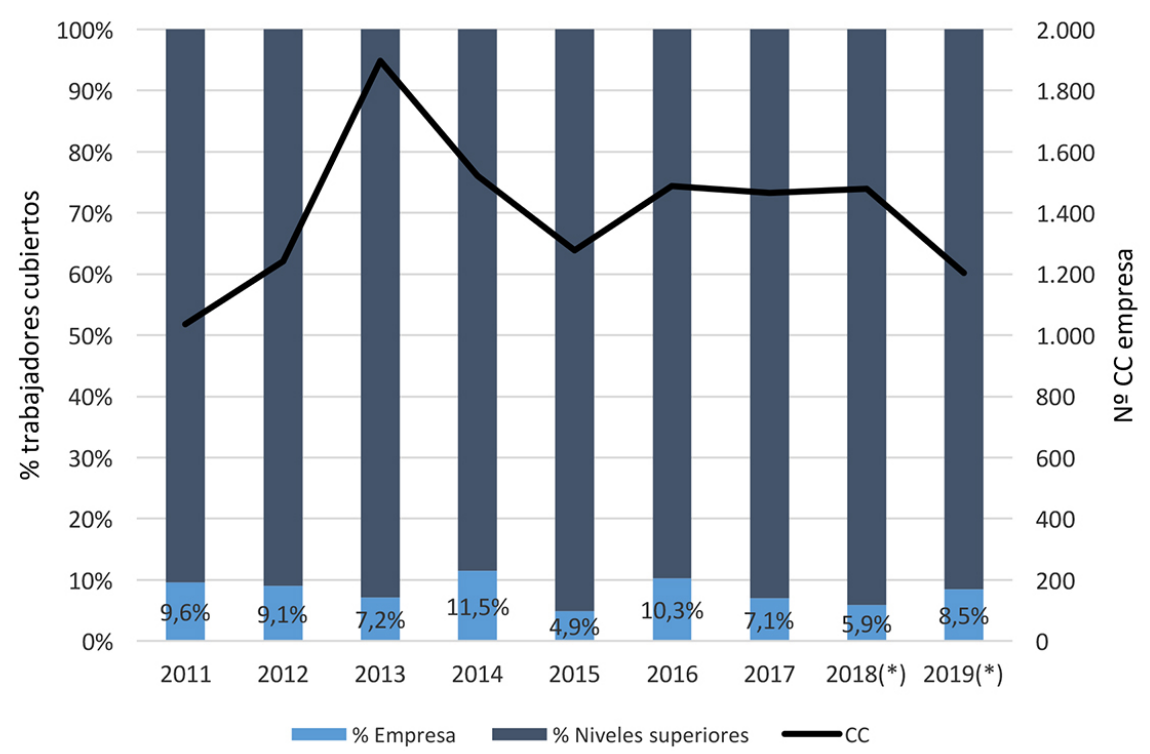

Gráfico 7. Número de convenios de empresa y porcentaje de trabajadores cubiertos según tipo de convenio. Fuente: Estadísticas del Ministerio de Trabajo. ${ }^{*}$ datos provisionales.

lectiva, son escasos. El gráfico 7 muestra la evolución experimentada entre 2011 y 2019. A pesar de que se aprecia un claro efecto de las reformas de 2011 y 2012, aumentando el número de convenios de empresa, parece que terminaron fracasando en cambiar la estructura de la negociación colecti$v^{18}$. Así, la situación se ha mantenido prácticamente inalterada: alrededor del $90 \%$ de los empleados están cubiertos por convenios colectivos sectoriales (especialmente los provinciales), mientras que los convenios de empresa apenas cubren el 10\%.

Varios son los motivos que explican esta pertinaz resistencia al cambio por parte de nuestra negociación colectiva. El primero está relacionado con la estructura productiva y es común a otros países de nuestro entorno. A pesar de las diferencias, todos los países comparten que sus economías están formadas principalmente por pequeñas y medianas empresas. Este es un claro obstáculo para el desarrollo de una negociación descentralizada por razones tanto legales como prácticas. Por un lado, la mayoría de los ordenamientos jurídicos no incluyen la representación de los empleados para todo tipo de empresas. Por otro, incluso cuando hay representantes, es posible que la empresa no tenga una cultura de negociación o, simplemente, que no le compense asumir los costes que de la misma se derivan cuando cuentan con un marco regulatorio a nivel sectorial que le cubre.

En segundo lugar, el contexto político y económico también tiene una clara influencia. La mayoría de las reformas adoptadas durante la crisis financiera fueron impuestas por los gobiernos de manera unilateral (y, en algunos casos, por las instituciones europeas o internacionales). Esto puede afectar la efectividad de las reformas en sí mismas, teniendo en cuenta que quienes están principalmente llamados a implementarlas no estuvieron involucrados en su creación. Además, a pesar de que la crisis puede funcionar como palanca de cambio, presionando para adoptar medidas que nunca se hubieran considerado en su ausencia, también es cierto que produce dificultades para aplicarlas o, simplemente, para introducir cambios en un contexto de arenas movedizas.

La última razón es el comportamiento estratégico de los interlocutores sociales. La resistencia a descentralizar la negociación colectiva se ha atribuido tradicionalmente a los sindicatos porque significa una clara reducción de su poder de negociación. Sin embargo, las empresas también pue-

\footnotetext{
${ }^{18}$ Muñoz Ruiz, A. B.; Pérez del Prado, D., "Negociación colectiva de empresa: ¿̨hacia la recuperación económica o aún en crisis?", Revista de información laboral, 10, 2014.
} 
den ser reacias a este tipo de cambios debido a varias razones: la descentralización de la negociación colectiva reduce la influencia de las asociaciones de empleadores en sus asociados y los incentivos para asociarse; esto también puede debilitar su posición ante el Gobierno y los sindicatos; y, finalmente puede afectar la competitividad dentro del mismo mercado, ya que elimina el mismo marco regulatorio para un sector o actividad específicos. Como consecuencia, los interlocutores sociales pueden desarrollar un comportamiento estratégico para evitar la implementación práctica de la descentralización, reduciendo o, incluso, obstaculizando su efectividad.

Sea como fuere, lo cierto es que tras décadas de intentos fallidos parece que el debate empieza a apartarse de cuál ha de ser la estructura para centrarse en el modo en que se ha de articular. Los informes más recientes de la OCDE señalan que «mientras muchos países de la OCDE han dado pasos hacia la descentralización en las últimas dos décadas, los estudios muestran que los mejores resultados en términos de empleo, productividad y salarios parecen alcanzarse cuando los convenios sectoriales establecen amplias condiciones marco, pero dejan disposiciones detalladas a nivel de empresa. Sin embargo, otras formas de descentralización que simplemente reemplazan sectorial con nivel de empresa la negociación, sin coordinación dentro y entre sectores, tiende a asociarse con resultados algo más pobres» ( $\mathrm{p}$ 135). A lo que se añade «los resultados muestran que los sistemas coordinados de negociación colectiva están asociados con un mayor empleo, menor desempleo, una mejor integración de los grupos vulnerables y menos desigualdad salarial que los sistemas descentralizados. Esto destaca el papel de la coordinación salarial como herramienta para garantizar que los acuerdos se establecen teniendo en cuenta sus efectos macroeconómicos sin socavar la competitividad del país, así como la contabilidad de la situación del ciclo económico» ${ }^{19}$.

Por consiguiente, lo importante no es si la estructura está más o menos centralizada o descentralizada, sino si se encuentra coordinada. En esto parece que tienen ventaja los sistemas en los que predomina el convenio sectorial, dada su capacidad de control "desde arriba", sin que con ello se excluya la existencia de modelos descentralizados bien coordinados. En suma, partiendo del modelo de negociación colectiva existente en cada país, los esfuerzos han de centrarse en lograr el mayor grado de coordinación posible.

¿Y qué hemos de entender por tal? «La coordinación se refiere al grado de qué jugadores menores siguen deliberadamente lo que deciden los grandes. La coordinación puede ocurrir entre unidades de negociación a diferentes niveles (por ejemplo, cuando los convenios a nivel sectorial o empresarial siguen las pautas fijadas por organizaciones de nivel superior o por un pacto social) o entre unidades al mismo nivel (por ejemplo, cuando algunos sectores o empresas siguen los estándares establecidos en otro sector / empresa). Muchos estudios han encontrado en diferentes prácticas de coordinación un factor principal detrás de la evolución de los salarios y flexibilidad macro, es decir, la capacidad de la economía para adaptarse a los shocks macroeconómicos» ${ }^{20}$.

¿Y qué hemos hecho en nuestro país en los últimos años? Pues exactamente todo lo contrario, al intentar descentralizar la negociación colectiva hemos reducido el grado de coordinación del conjunto del sistema, tal y como muestra la siguiente tabla. Como puede comprobarse, España pasó de ser un país predominantemente centralizado y coordinado a predominantemente centralizado y débilmente coordinado. En un primer momento, esto se debió a la falta de acuerdo para la negociación colectiva en 2009, pero luego esta situación se ha mantenido a pesar de la firma de acuerdos en 2010, 2012 y 2015, lo que evidenciaría el efecto permanente de las reformas.

\footnotetext{
${ }^{19}$ OECD, Negotiating our way up: collective bargaining in a changing world of work., 2019, P. I 35 . En general, sobre este asunto, léase el capítulo 3 de este informe. Traducción propia.

${ }^{20} \mathrm{Ibid}$., p. 61. Traducción propia.
} 
Tabla 1. Taxonomía de la negociación colectiva.

\begin{tabular}{|l|l|l|l|l|l|l|l|l|l|l|l|l|l|l|l|l|}
\hline & $\mathbf{2 0 0 0}$ & $\mathbf{2 0 0 1}$ & $\mathbf{2 0 0 2}$ & $\mathbf{2 0 0 3}$ & $\mathbf{2 0 0 4}$ & $\mathbf{2 0 0 5}$ & $\mathbf{2 0 0 6}$ & $\mathbf{2 0 0 7}$ & $\mathbf{2 0 0 8}$ & $\mathbf{2 0 0 9}$ & $\mathbf{2 0 1 0}$ & $\mathbf{2 0 1 1}$ & $\mathbf{2 0 1 2}$ & $\mathbf{2 0 1 3}$ & $\mathbf{2 0 1 4}$ & $\mathbf{2 0 1 5}$ \\
\hline Bélgica & PCC & PCC & PCC & PCC & PCC & PCC & PCC & PCC & PCC & PCC & PCC & PCC & PCC & PCC & PCC & PCC \\
\hline Francia & PCW & PCW & PCW & PCW & PCW & PCW & PCW & PCW & PCW & PCW & PCW & PCW & PCW & PCW & PCW & PCW \\
\hline Italia & PCW & PCW & PCW & PCW & PCW & PCW & PCW & PCW & PCW & PCW & PCW & PCW & PCW & PCW & PCW & PCW \\
\hline Portugal & PCW & PCW & PCW & PCW & PCW & PCW & PCW & PCW & PCW & PCW & PCW & PCW & PCW & PCW & PCW & PCW \\
\hline España & PCW & PCW & PCC & PCC & PCC & PCC & PCC & PCC & PCC & PCW & PCW & PCW & PCW & PCW & PCW & PCW \\
\hline
\end{tabular}

Fuente: OCDE, 2019. Leyenda: PCW. Predominantly Centralised and Weakly co-ordinated; PCC. Predominantly Centralised and Co-ordinated.

De ahí la importancia de prestar atención a este otro elemento de la coordinación entre niveles, incluso más si cabe que a la estructura. En este sentido, la revisión de la última reforma laboral que, a juzgar por los datos, parece necesaria, debiera pivotar precisamente en asegurar un sistema bien coordinado.

\section{La recuperación de los salarios}

Otra de las áreas que, sin lugar a dudas, ha de ser objeto de atención son los salarios. A pesar de que a primera vista lo que pudiera pensarse es que la actuación en este campo debiera estar dirigida a compensar los efectos de la Gran Recesión, lo cierto es que las tendencias de política salarial vienen de largo, la trasciende, lo que exige actuaciones de carácter estructural.

En efecto, no debiera permitirse que el árbol de la crisis no nos deje ver el bosque de la comprensión salarial $^{21}$. No hay lugar a dudas de que el salario ha jugado un papel central en la política económica seguida durante la segunda parte de la Gran Recesión, la llamada Crisis del euro, como mecanismo de devaluación interna. Como se ha señalado y muestra el siguiente gráfico, si los salarios se hubieran acompasado a la productividad, deberían haber acumulado un crecimiento del $10,31 \%$ y el $8,85 \%$ respectivamente. Sin embargo, la realidad es que el coste real del trabajo ha ex-

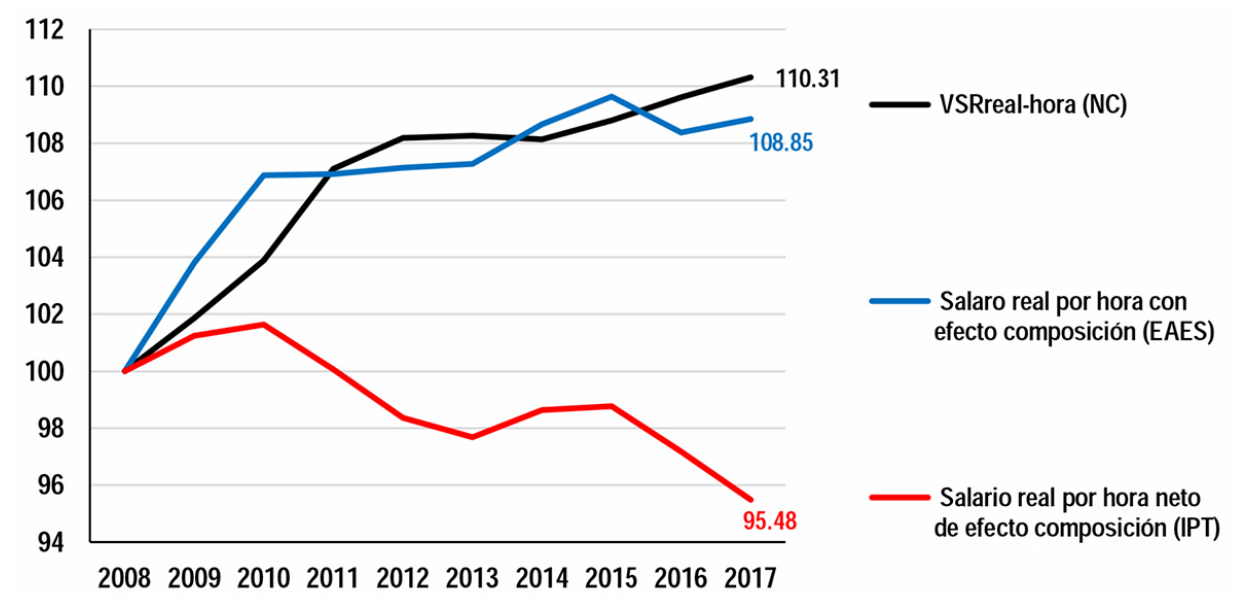

Gráfico 8. Evolución de los costes laborales.

Fuente: Gómez Rufián, 2019.

${ }^{21}$ Mejor que el más adecuado, aunque con naturaleza más eufemística, de "moderación salarial”. 
perimentado una caída, en esos términos acumulados, del -4,52\%. «En otras palabras, las empresas españolas, frente al discurso tradicionalmente dominante, y sobre el que luego se realizarán ciertas reflexiones, desembolsan en términos reales, casi diez años después de iniciarse la recesión, alrededor de cinco puntos menos en costes reales por el factor trabajo que poseen ${ }^{22}$.

Esto ha producido un efecto claro en el poder adquisitivo y bienestar social de buena parte de la población. En el mejor de los casos, empleando el incremento salarial medio pactado en los convenios colectivos en España, la ganancia de poder adquisitivo se habría situado entre la congelación y el 1\% para el período 2008-2014. Sin embargo, estudios como el anteriormente reseńado y del que se ha obtenido también el siguiente gráfico, muestran que la situación podría ser incluso peor, arrojando una caída acumulada del poder adquisitivo del $-10,83 \%$, aunque aquí la franja temporal es algo más larga y llega hasta 2017.

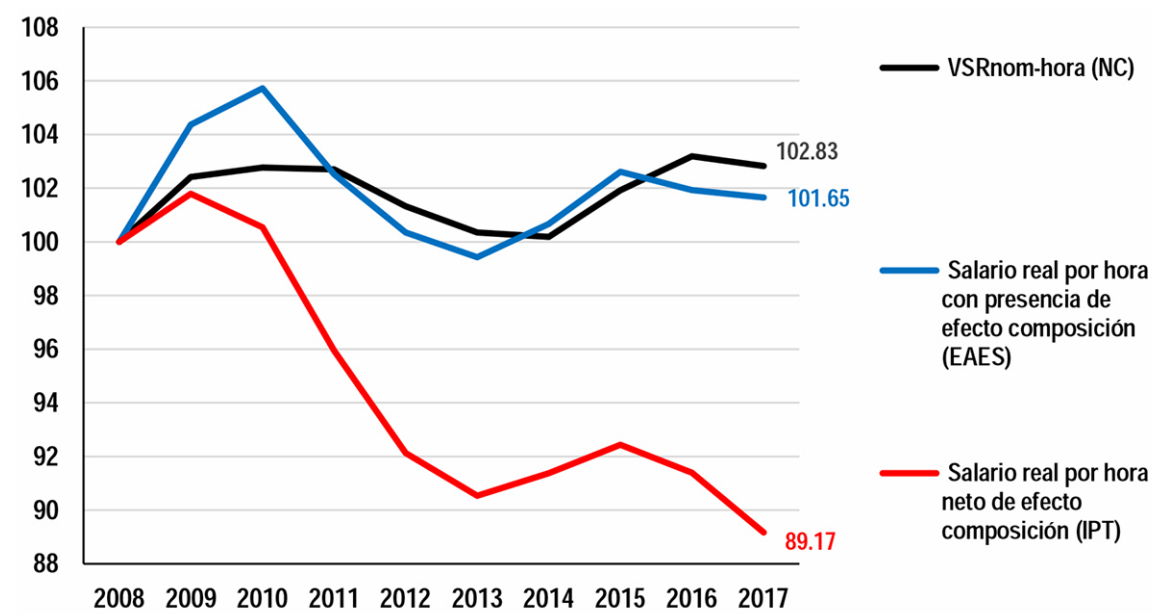

Gráfico 9. Evolución del poder adquisitivo.

Fuente: Gómez Rufián, 2019.

Sin embargo, al margen del efecto de la Gran Recesión, los datos nos permiten ver que la compresión salarial no es una cuestión de la crisis o de las crisis, sino que reviste naturaleza estructural. Como se ha señalado «el descenso de la participación de las rentas del trabajo en la renta nacional desde al menos los ańos 80 constituye una tendencia global de carácter estructural ampliamente contrastada, y no solo en las economías desarrolladas. Esto significa que en las últimas décadas el salario real medio ha crecido sistemáticamente por debajo de la productividad, mostrando ambas variables una tendencia al desacoplamiento macroeconómico a largo plazo» ${ }^{23}$.

El Gráfico 10 evidencia bien a las claras esta tendencia estructural de los mercados de trabajo en general y del nuestro en particular. En él se muestra la participación de las rentas del trabajo en el conjunto del PIB, tomando como referencia el que había en 1978. Pues bien, en tres décadas, tal contribución se habría reducido en prácticamente un $20 \%$ en nuestro país, lo que como es lógico no solamente tiene efectos sobre las personas trabajadoras, sino sobre el conjunto de la economía dada su incidencia sobre la equidad y, de esta, sobre la eficiencia ${ }^{24}$.

\footnotetext{
${ }^{22}$ Gómez Rufí́n, L., "Evolución de la dinámica salarial en el periodo 2008-2017 según el Índice de Precios del Trabajo: salarios nominales y reales, derivas salariales y correlación el salario - ciclo económico", Revista Aranzadi Doctrinal, 11, 2019, Aranzadi Thomson Reuters.

${ }^{23}$ Vizán Rodríguez, C., La caída del peso económico de las rentas del trabajo, Fundación Alternativas, 2018, p. 2, fecha de consulta 15 febrero 2021, en https://www.fundacionalternativas.org/laboratorio/documentos/zoom-economico/la-caida-del-pesoeconomico-de-las-rentas-del-trabajo.

${ }^{24}$ Piketty, T., Capital e ideología, Deusto, Barcelona, 2019.
} 


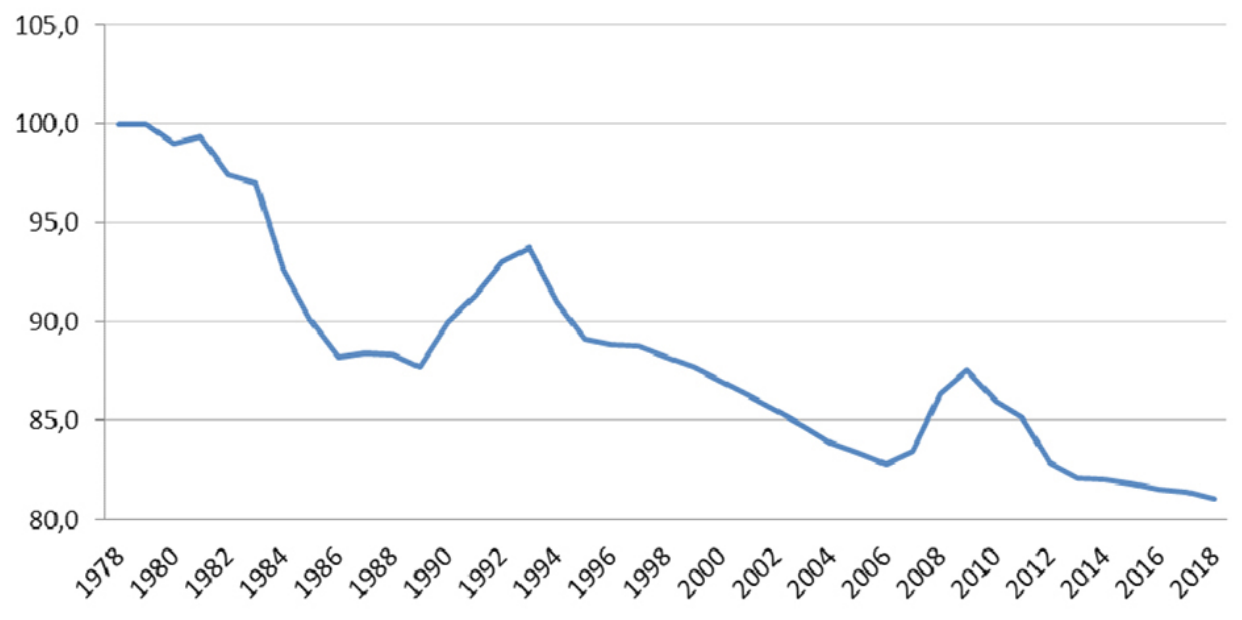

Gráfico 10. Participación ajustada de las rentas del trabajo.

Fuente: Vizán Rodríguez, 2018. Remuneración por asalariado/ PIB a precios de mercado por ocupado. Índice: 1978/100.

\section{Políticas de empleo para un nuevo Estado del Bienestar}

Finalmente, la reforma del mercado de trabajo ha de tener muy en cuenta las políticas de empleo como mecanismo que propicia que funcione más eficientemente. A este respecto, debe tenerse presente que este tipo de políticas no crean por sí mismas empleo. Lo que genera empleo es la demanda agregada, el funcionamiento general de la economía. Sin embargo, este tipo de políticas pueden resultar muy beneficiosas en lo que hace a alcanzar un objetivo paralelo y complementario, que el mercado de trabajo y, por ende, el conjunto de la economía, funcionen mejor.

Pues bien, tal y como muestra el siguiente gráfico, el nivel de gasto como porcentaje del PIB de nuestro país es relativamente bueno, pues se encuentra por encima de la media europea, incluso después del tremendo impacto que tuvo la crisis financiera sobre las finanzas públicas. Es cierto que

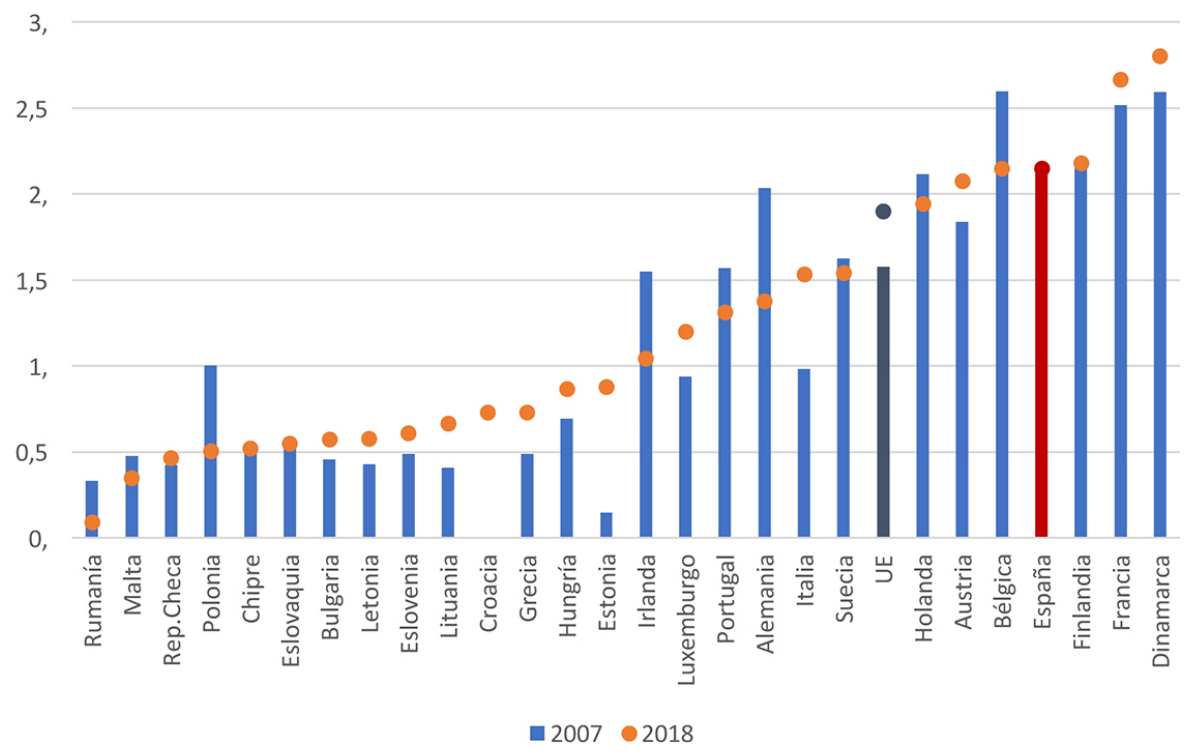

Gráfico 11. Gasto en políticas de empleo como porcentaje del PIB.

Fuente: elaboración propia a partir de Eurostat. 
la situación cambia si empleamos otras variables como el gasto por persona desempleada, dadas las elevadas tasas de paro que aún padecemos, pero el punto de partida, al menos desde la perspectiva del gasto, no es del todo malo.

Si vamos al detalle, podemos observar que la mayor parte del gasto se destina a la protección por desempleo. Tradicionalmente se ha analizado este tipo de resultados como algo negativo, señalándose que lo deseable sería su traspaso hacia las políticas activas. Esto es sólo parcialmente cierto, pues parte de la consideración del fenómeno del paro como algo enteramente controlable, cuando esto no tiene que ser necesariamente así, por ejemplo, cuando los incrementos provienen de una crisis económica. El mal dato no es que se gaste mucho en desempleo, sino que el desempleo estructural sea relativamente alto. Lo que hay que corregir no es el gasto, sino aquello que impide que el paro sea menor.

Por lo demás, desde el nacimiento de los Estados del bienestar, el fenómeno del desempleo ha llevado aparejado la provisión de un determinado nivel de protección social, lo que se ha considerado tradicionalmente necesario tanto desde la perspectiva de la equidad, como de la eficiencia ${ }^{25}$. De ahí que, al margen de modificaciones, no menores, de carácter técnico, especialmente en el lado de la protección asistencial, de la compatibilidad del trabajo con la protección por desempleo y de la reordenación y coordinación con otras formas de protección social, especialmente tras el nacimiento del ingreso mínimo vital; la reforma de las mal llamadas políticas pasivas debiera dirigirse a su consideración plena como un derecho a la protección social en caso de desempleo. El modelo de Estado sancionador y persona desempleada bajo sospecha ha de ser superado para sustituirlo por otro más avanzado en el que predomine su consideración como derecho y en el que los mecanismos de control y sanción, como en cualquier otro, no sean la nota predominante.

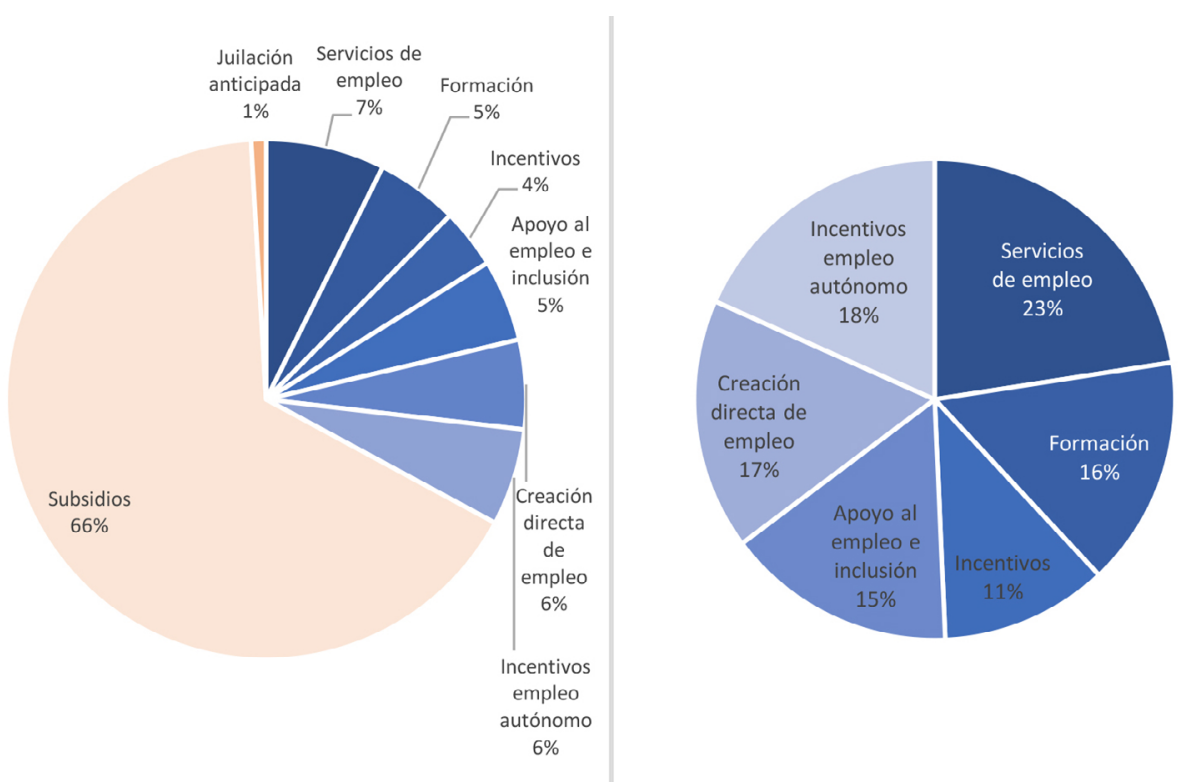

Gráfico 11. Gasto en políticas de empleo como porcentaje del PIB.

Fuente: elaboración propia a partir de Eurostat.

Lo mismo ocurre con las mal llamadas políticas activas, que según el gráfico señalado suponen un tercio del gasto total en políticas de empleo. No solamente resulta necesario reorientar el gasto hacia aquellas que ofrezcan mejores resultados, para lo cual la evaluación ha de convertirse en un

${ }^{25}$ Pérez del Prado, D., Prestación por desempleo: intensidad, duración y control; los efectos económicos de la protección frente al paro, Lex Nova, Valladolid, 2014. 
elemento estructural de su gestión; sino que se ha de partir de su reconsideración como un auténtico derecho de ciudadanía y, por consiguiente, como una prestación más del Estado del bienestar. Desde esta perspectiva, la clave no reside en activar a la persona desempleada, que en la mayoría de las ocasiones no va a encontrar empleo, no porque no quiera, sino porque no lo hay en atención a sus circunstancias y cualificaciones; sino en activar los servicios que se proveen, adaptándolos a las necesidades de las personas (desempleadas, pero también ocupadas), de las empresas y del conjunto de la economía.

\section{A modo de conclusiones}

A lo largo de estas páginas hemos tenido ocasión de analizar buena parte de los problemas más relevantes de nuestro mercado de trabajo, aportando datos adicionales para un debate que resulta impostergable. Buena parte los problemas destacados nos han acompañado durante décadas, el resto simplemente revisten una nueva apariencia.

Como se indica en el artículo que sirve de pretexto al presente trabajo, es tiempo para que estas reformas arranquen de un nuevo pacto social. Varios son los motivos que lo explican. En primer lugar y, como acabamos de seńalar, lo enquistado de buena parte de las deficiencias e ineficiencias que arrastra nuestro mercado laboral. En segundo lugar, también es preciso tener en cuenta un componente coyuntural no menor, la enorme huella dejada por las dos crisis económicas y sociales, la pasada y la actual, sobre el conjunto de la sociedad espańola. En tercer lugar, junto a lo anterior, también es preciso tomar en consideración un factor de fondo o estructural innegable, como es el relativo a la transformación disruptiva que la digitalización supone. Por último, resulta evidente que las soluciones coyunturales no han arrojado los resultados esperados.

Teniendo presente todas estas variables, es tiempo de afrontar los problemas tradicionales de nuestro mercado laboral desde una perspectiva renovada y, lo que es más importante, con unos cimientos lo suficientemente robustos como para, no solamente resolverlos de una vez por todas, sino para sentar las bases de un mercado de trabajo más justo, sostenible y equitativo.

\section{Bibliografía}

ASEMPLEO, El monográfico del Mercado Laboral, Asempleo, 2020, fecha de consulta 15 febrero 2021, en https://asempleo.com/wp-content/uploads/2020/10/Bolet\%C3\%ADn-AMLn\%C2\%BA-159-Octubre-2020-monografico.pdf.

BENTOLILA, S.; SAINT-PAUL, G., "The macroeconomic impact of flexible labor contracts, with an application to Spain", CEMFI, DT, n. ${ }^{\circ}$ No. 9106, 1991.

DE LA RICA, S., "La penalización salarial de la temporalidad: ¿Qué efectos tiene en las decisiones familiares?", en Propuesta para la reactivación laboral en España, Fedea, 2010.

DURÁN, F., Informe sobre los riesgos laborales y su prevención. La seguridad y salud en el trabajo en España, Presidencia del Gobierno, Madrid, 2001.

EUROPEAN PARLIAMENT, Precarious Employment in Europe: Patterns, Trends and Policy Strategies, European, Brussels, 2017.

GÓMEZ RUFIÁN, L., "Evolución de la dinámica salarial en el periodo 2008-2017 según el Índice de Precios del Trabajo: salarios nominales y reales, derivas salariales y correlación el salario - ciclo económico", Revista Aranzadi Doctrinal, n.o 11, 2019, Aranzadi Thomson Reuters, p. 16.

HERRANZ MARTÍN, V.; TOHARIA CORTÉS, L., "Do temporary contracts increase work accidents? A microeconomic comparison between Italy and Spain”, Fedea DT, n.o No. 10, 2003. 
JIMENO, J. F.; TOHARIA, L., "The effects of fixed-term employment on wages: theory and evidence from Spain”, Investigaciones Economicas, vol. 17, n.o 3, 1993, Fundación SEPI, pp. 475-494.

MERCADER UGUINA, J. R., El futuro del trabajo en la era de la digitalización y la robótica, Tirant lo Blanch, Valencia, 2017.

MERCADER UGUINA, J. R., Estructura de la negociación colectiva y relaciones entre convenios, Editorial Civitas : Ediciones de la Universidad Autónoma de Madrid, Madrid, 1994.

MERCADER UGUINA, J. R., "La empresa como nuevo centro de gravedad de la estructura de la negociación colectiva: la reforma de la Ley 3/2012”, en García-Perrote Escartín, I., Mercader Uguina, J. R. (eds.) La regulación del mercado laboral: un análisis de la Ley 3/2012 y de los aspectos laborales del Real Decreto-ley 20/2012), Lex Nova, Valladolid, 2012.

MUÑOZ RUIZ, A. B.; PÉREZ DEL PRADO, D., "Negociación colectiva de empresa: ¿̨hacia la recuperación económica o aún en crisis?”, Revista de información laboral, n.o 10, 2014, pp. 15-40.

NIETO ROJAS, P., "Acción colectiva en las plataformas digitales. ¿sindicatos tradicionales y movimientos de base para representar idénticos intereses?", en El futuro del trabajo: cien años de la OIT. XXIX Congreso Anual de la Asociación Española de Derecho del Trabajo y de la Seguridad Social, Ministerio de Trabajo y Seguridad Social, Madrid, 2019 (Informes y estudios).

OECD, Collective bargaining in a changing world of work, OECD, Geneva, 2017, pp. 125-186, fecha de consulta 17 febrero 2021, en https://www.oecd-ilibrary.org/employment/oecdemployment-outlook-2017/collective-bargaining-in-a-changing-world-of-work_empl_outlook-2017-8-en.

OECD, Negotiating our way up: collective bargaining in a changing world of work., 2019.

PÉREZ DEL PRADO, D., "Los efectos de la temporalidad más allá del mercado de trabajo", en Gómez Abelleira, F. J. (ed.) La temporalidad laboral como problema jurídico: diagnósticos y soluciones, Tirant lo Blanch, 2014, pp. 61-96.

PÉREZ DEL PRADO, D., Prestación por desempleo: intensidad, duración y control ; los efectos económicos de la protección frente al paro, Lex Nova, Valladolid, 2014.

PIKETTY, T., Capital e ideologia, Deusto, Barcelona, 2019.

RODRÍGUEZ FERNÁNDEZ, M. L., "Calificación jurídica de la relación que une a los prestadores de servicios con las plataformas digitales", en Plataformas digitales y mercado de trabajo, Subdirección General de Información Administrativa y Publicaciones, 2018, pp. 57-89.

URZÍ BRANCATI, M.C; PESOLE, A; FERNÁNDEZ-MACÍAS, E., New evidence on platform workers in Europe: results from the second COLLEEM survey., European Commission, Directorate-General for Economic and Financial Affairs, Brussels, 2020.

VIZÁN RODRÍGUEZ, C., La caída del peso económico de las rentas del trabajo, Fundación Alternativas, 2018, fecha de consulta 15 febrero 2021, en https://www.fundacionalternativas.org/ laboratorio/documentos/zoom-economico/la-caida-del-peso-economico-de-las-rentas-deltrabajo. 